\title{
THE LONG-BASELINE NEUTRINO EXPERIMENT IN THE US
}

\section{MILIND V. DIWAN}

Physics Department, Brookhaven National Laboratory

Upton, NY 11973, USA

E-mail: diwan@bn $1.90 \mathrm{~V}$

\begin{abstract}
We report on the status of the Long-baseline Neutrino Experiment (LBNE) and the physics potential of the LBNE research program. LBNE (Long-Baseline Neutrino Experiment) is an accelerator-based neutrino oscillation experiment. LBNE will produce a muon-neutrino beam using protons from Fermilab's Main Injector and will detect electron-neutrino appearance and muon-neutrino disappearance using a Liquid Argon TPC located at a distance of $1300 \mathrm{~km}$ at the Sanford Underground Research Facility in South Dakota. The primary physics motivation of LBNE is to determine the neutrino mass hierarchy, to determine the octant of the neutrino mixing angle $\theta_{23}$, to search for $\mathrm{CP}$ violation in neutrino oscillation, and ultimately, to precisely measure the size of any CP-violating effect that is discovered. LBNE is envisioned as a phased program with international participation and leadership. The first phase of the experiment is currently in the detailed-design stage which includes engineering design of the neutrino beamline and underground caverns for the far and near detectors.
\end{abstract}

XV Workshop on Neutrino Telescopes,

11-15 March 2013

Venice, Italy 


\section{Introduction}

In the current picture of neutrino oscillations, three flavors of neutrinos are related to three mass states by the Pontecorvo-Maki-Nakagawa-Sakata mixing matrix [1,2]. The mixing can be described by two $\Delta m^{2}$ parameters $\left(\left|\Delta m_{32}^{2}\right|,\left|\Delta m_{21}^{2}\right|\right)$, three mixing angles $\left(\theta_{23}, \theta_{12}\right.$, and $\left.\theta_{13}\right)$, which have all been measured and a $\mathrm{CP}$ violating phase $\left(\delta_{c p}\right)$ which is unknown[3]. The oscillation phenomena naturally falls into two domains: atmospheric neutrino oscillations, and Solar neutrino oscillations [3]. Measurement of a value of $\delta_{C P}$ not equal to zero or $\pi$ would be the first observation of CP violation in the lepton sector. The measured value of $\theta_{23}$ is near to $45^{\circ}$; if it is exactly $45^{\circ}$, which would indicate that $v_{\mu}$ and $v_{\tau}$ have equal contributions from $v_{3}$, then that could be evidence for a previously unknown symmetry. The $\theta_{23}$ octant, i.e., whether $\theta_{23}$ is less than, greater than, or equal to $45^{\circ}$, is unknown. The differences between the mass states, $\Delta m_{21}^{2}$ and $\left|\Delta m_{32}^{2}\right|$, have also been measured, though the true mass hierarchy is unknown. The case in which $\Delta m_{32}^{2}$ is greater than zero is referred to as "normal hierarchy" (NH) and the opposite case is called "inverted hierarchy" (IH). Determination of the unknowns in neutrino mixing needs further experiments in the atmospheric oscillation domain of $\left|\Delta m_{32}^{2}\right|$ for conversion of muon and electron type neutrinos into each other.

Since the mixing angle $\theta_{13}$ is now known to be non-zero[4, 5, 6, 7, 8, 9], it is possible to probe neutrino oscillations via the appearance of electron neutrinos in a muon neutrino beam. The appearance probability is

$$
\begin{aligned}
P\left(v_{\mu} \rightarrow v_{e}\right) \approx & \sin ^{2} \theta_{23} \frac{\sin ^{2} 2 \theta_{13}}{(\hat{A}-1)^{2}} \sin ^{2}((\hat{A}-1) \Delta) \\
& +\alpha \frac{\sin \delta_{C P} \cos \theta_{13} \sin 2 \theta_{12} \sin 2 \theta_{13} \sin 2 \theta_{23}}{\hat{A}(1-\hat{A})} \sin (\Delta) \sin (\hat{A} \Delta) \sin ((1-\hat{A}) \Delta) \\
& +\alpha \frac{\cos \delta_{C P} \cos \theta_{13} \sin 2 \theta_{12} \sin 2 \theta_{13} \sin 2 \theta_{23}}{\hat{A}(1-\hat{A})} \cos (\Delta) \sin (\hat{A} \Delta) \sin ((1-\hat{A}) \Delta) \\
& +\alpha^{2} \frac{\cos ^{2} \theta_{23} \sin ^{2} 2 \theta_{12}}{\hat{A}^{2}} \sin ^{2}(\hat{A} \Delta)
\end{aligned}
$$

In this equation $\alpha=\Delta m_{21}^{2} / \Delta m_{31}^{2}, \Delta=\Delta m_{31}^{2} L / 4 E, \hat{A}=2 V E / \Delta m_{31}^{2}, V=\sqrt{2} G_{F} n_{e} . n_{e}$ is the density of electrons in the Earth. Recall that $\Delta m_{31}^{2}=\Delta m_{32}^{2}+\Delta m_{21}^{2}$. Also notice that $\hat{A} \Delta$, which has absolute value of $L G_{F} n_{e} / \sqrt{2}$, is sensitive to the sign of $\Delta m_{31}^{2}$. Figure 1 shows the $v_{e}$ appearance probability at $1300 \mathrm{~km}$ for various values of $\delta_{C P}$. The value of $\sin ^{2}\left(2 \theta_{13}\right)$ determines the size of the $v_{e}$-appearance sample, the value of $\delta_{C P}$ affects the amplitude of the oscillation, the value of $\Delta_{31}$ affects the frequency of the oscillation, the mass hierarchy affects both the amplitude and frequency of the oscillation, and the value of $\theta_{23}$ enters unambiguously. Therefore $v_{e}$ appearance is sensitive to $\delta_{C P}$, the $\theta_{23}$ octant, and the mass hierarchy, as well as matter effects. For $\bar{v}_{e}$ appearance the $\mathrm{CP}$ phase and the matter potential change sign, and so a measurement of asymmetries between neutrinos and antineutrinos is sensitive to these parameters.

Figure 2 compares the neutrino-antineutrino asymmetries for different baselines. The asymmetry due to the matter effect, arising from the additional potential experienced by neutrinos and antineutrinos as they propagate in matter and entering Eq. 1.1 as $\hat{A} \Delta$, dominates the CP asymmetry 


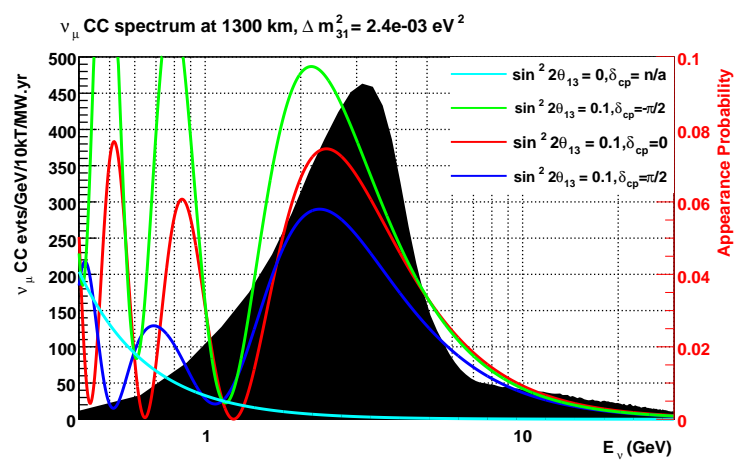

Figure 1: $v_{e}$ appearance probability at a distance of $1300 \mathrm{~km}$ as a function of energy. The green (red,blue) curve indicates a value of $\delta_{C P}=-\pi / 2(0, \pi / 2)$. The cyan curve shows the appearance probability if $\sin ^{2}\left(2 \theta_{13}\right)$ were zero. The black histogram shows the unoscillated $v_{\mu}$ spectrum. The spectrum is wellmatched to the first oscillation maximum.

at long baselines. In comparing the asymmetries for baselines of $290 \mathrm{~km}$ and $1300 \mathrm{~km}$ (right panel of Fig. 2), it is clear that for the first oscillation node, at $290 \mathrm{~km}$, there are degeneracies in which the value of $\delta_{C P}$ might be very different for $\mathrm{NH}$ and IH given the same asymmetry measurement. These degeneracies are not present at or above $\sim 1000 \mathrm{~km}$; this allows a long-baseline experiment to both determine the mass hierarchy and measure the CP phase with sufficient statistics. At baselines $>2500 \mathrm{~km}$, the matter asymmetries (see Fig. 2 left) overwhelm the CP asymmetry and lead to poor statistics in the matter suppressed running mode, neutrinos for $\mathrm{IH}$ and antineutrinos for $\mathrm{NH}$. Events near the second oscillation node (see Fig. 2 right) can be used to strengthen the degeneracy resolution or the $\mathrm{CP}$ measurement, but the statistics at the second oscillation node tend to be poor regardless of the baseline as long as it is above $\sim 1300 \mathrm{~km}$.

\section{The Long-Baseline Neutrino Experiment}

The Long-Baseline Neutrino Experiment (LBNE) will consist of a muon-neutrino beam from Fermilab to Sanford Underground Research Facility (SURF), a baseline of $1300 \mathrm{~km}$, and a massive underground far detector. The experiment will make use of protons from Fermilab's Main Injector and a new muon-neutrino beam line will be constructed. The far detector will be a liquid argon time projection chamber (LAr TPC) with an integrated photon detection system. SURF is located in western South Dakota and is an active underground research lab, currently housing the LUX and MAJORANA experiments. At the time of this presentation, the LBNE collaboration consisted of about 370 members at 61 institutions. Since that time, the collaboration has grown significantly with the addition of sixteen new collaborating institutions from outside the U.S.

LBNE experimental design consists of a $35 \mathrm{kt}$ LArTPC far detector at SURF, a capable high resolution near detector, and a neutrino beam with initial capability of $700 \mathrm{~kW}$ beam power, upgradable to $>2.3 \mathrm{MW}$. LBNE10 refers to the first phase of the design that currently has funding approval from the U.S. Department of Energy: a 10-kt LAr TPC and a neutrino beam with 700-kW 120$\mathrm{GeV}$ proton beam. The funding from the US has assured flexibility so that non-US collaboration partners can enhance the scope of the first phase while maintaining the overall schedule. Higher proton beam power at lower energies and upgrades to the design of the neutrino beam line both 
CP asymmetries in $v_{\mu} \rightarrow v_{e}$ at $1^{\text {st }}$ osc. node

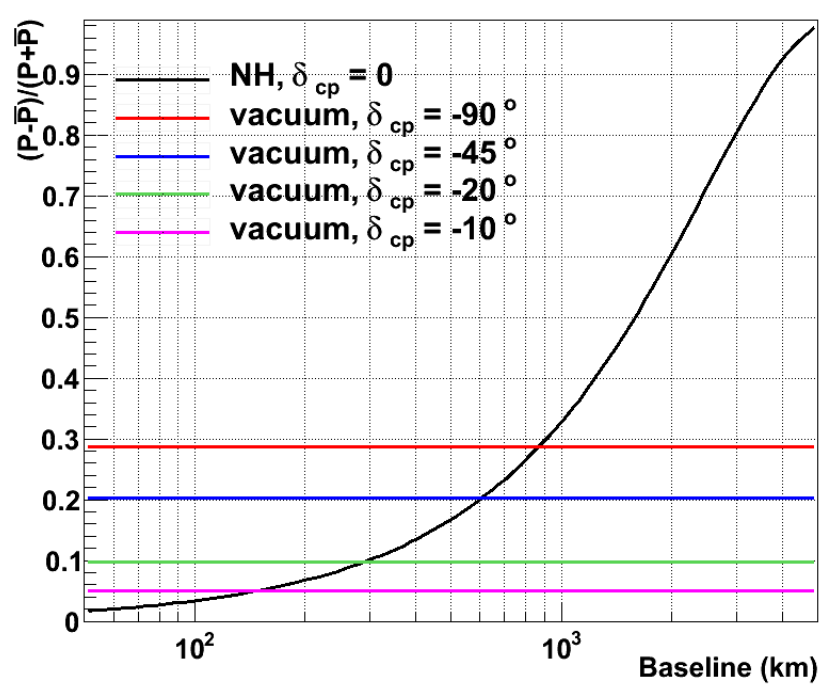

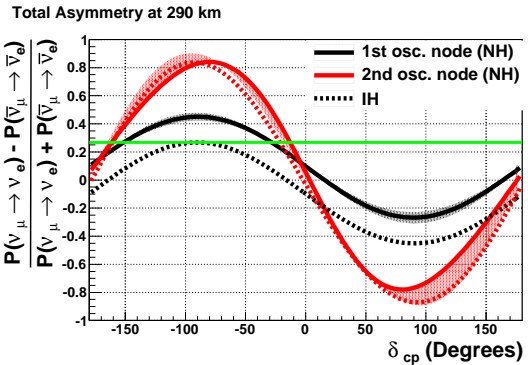

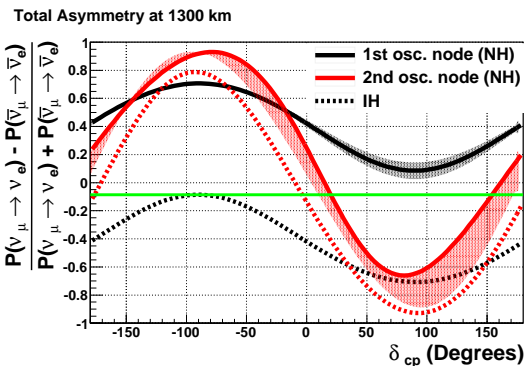

Figure 2: Left: Neutrino-antineutrino asymmetry in $v_{\mu} \rightarrow v_{e}$ appearance probability as a function of baseline at the first oscillation node. Right: Neutrino-antineutrino asymmetry as a function of $\delta_{C P}$ for the first and second oscillation nodes at baselines of $290 \mathrm{~km}$ (top) and $1300 \mathrm{~km}$ (bottom).

provide increased sensitivity. A larger mass underground far detector will enable greater physics reach and scientific breadth to the experiment.

Figure 3 shows the sensitivity of LBNE10 to the determination of the mass hierarchy and the discovery of $\mathrm{CP}$ violation. The running conditions are assumed to be $5 \mathrm{yrs}$ of running in each polarity (neutrino and antineutrinos) with $700 \mathrm{~kW}$ of beam power; however some differences in beam conditions are possible including running with 120 or $80 \mathrm{GeV}$ proton beam. LBNE10 represents a significant step forward in sensitivity relative to current experiments; in combination with NOvA and T2K, LBNE10 has an expected average significance of better than $3 \sigma$ to determine the mass hierarchy for all values of $\delta_{C P}$, and can find evidence of CP violation at $\sim 3 \sigma$ level for a significant fraction of the phase space. For these estimates $\sigma$ is simply $\sqrt{\Delta \chi^{2}}$. Figure 4 shows the same sensitivity for the full scope of LBNE with $35 \mathrm{kt}$ detector with the same running conditions. The left panel of Fig. 5 shows the ability of LBNE10 to determine the $\theta_{23}$ octant as a function of the true value of $\theta_{23}$. The sensitivities presented here are calculated using GLoBES[10, 11] and are described in greater detail in [12].

The experimental sensitivity becomes more significant with the full scope of LBNE with a $35 \mathrm{kt}$ detector (as in Fig. 4) and higher beam power. Figure 5 (right panel) and Fig. 6 show the increase in sensitivity that will be possible with a higher power beam and a larger far detector in one possible staging scenario. In these plots, the three levels of exposure correspond to LBNE10 and the first and second phases of increased detector mass and beam intensity [13, 14].

Observation of $v_{e}$ appearance in atmospheric neutrinos, which requires that the detector be underground, provides additional sensitivity to neutrino oscillation parameters, particularly the mass hierarchy. The $\mathrm{MH}$ sensitivity from atmospheric neutrinos is largely independent of the true value of $\delta_{C P}$. At $\delta_{C P} \approx \pi / 2(-\pi / 2)$ in $\mathrm{NH}(\mathrm{IH})$, the sensitivity from atmospheric neutrinos is approxi- 

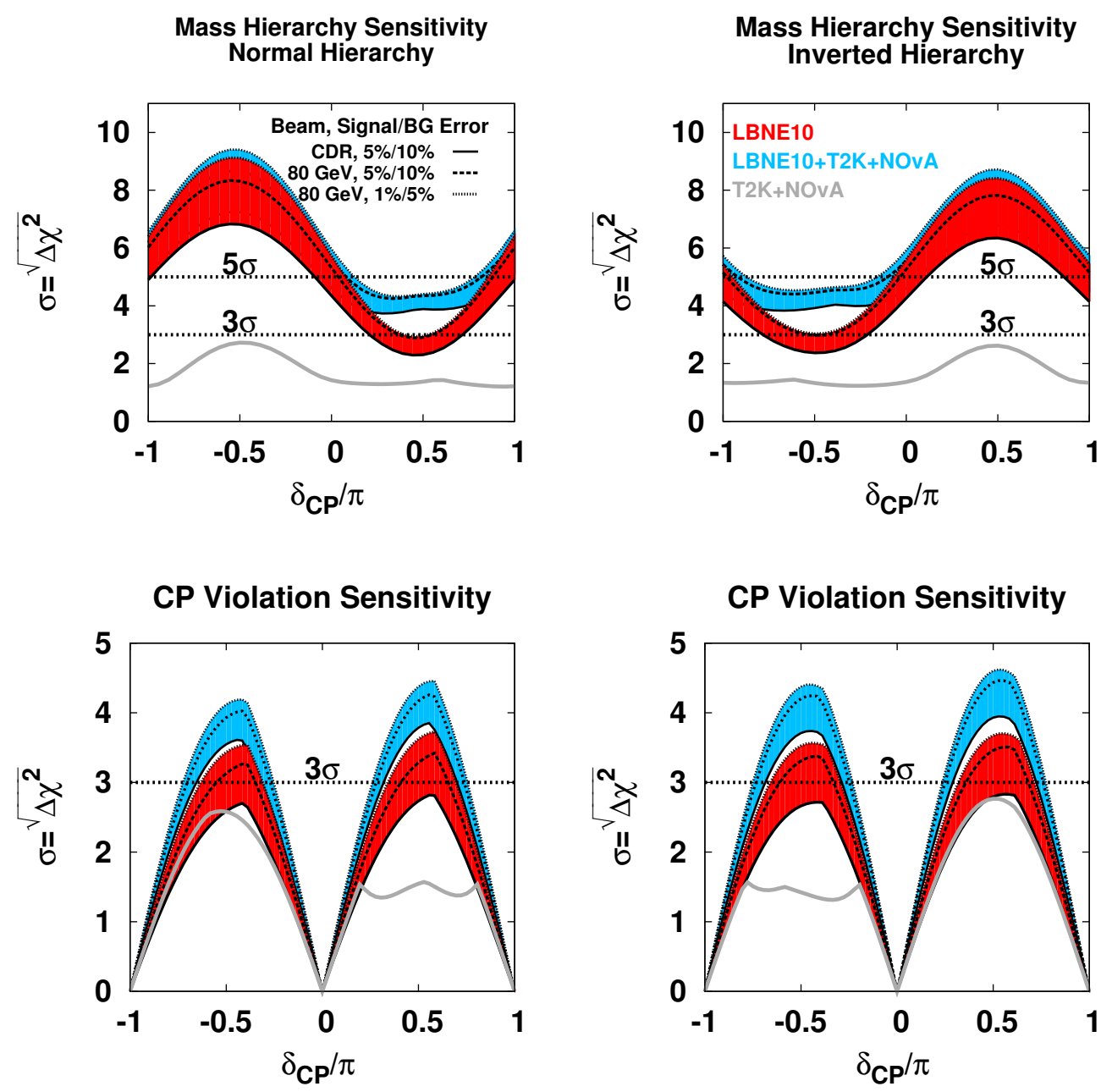

Figure 3: Sensitivity of LBNE10 to determination of mass hierarchy (top) and discovery of CP violation (bottom) for $\mathrm{NH}$ (left) and $\mathrm{IH}$ (right). The bands represent a range of possible sensitivities from variations in beam design and assumptions about the uncertainty of signal and background normalizations. NOvA and $\mathrm{T} 2 \mathrm{~K}$ are shown in grey, LBNE10 in red, and all three combined in blue.

mately equal to that from the beam data, and the systematic uncertainties are largely independent. Other physics topics that are possible with an underground far detector include proton decay, particularly the $p \rightarrow v K^{+}$decay mode, and the detection of supernova burst neutrinos if there is a galactic supernova during the lifetime of the experiment.

\section{Summary}

The 1300-km baseline between FNAL and SURF provides the opportunity to measure the remaining unknown neutrino oscillation parameters using $v_{\mu} \rightarrow v_{e}$ oscillations. LBNE is envisioned to have a $35 \mathrm{kt}$ underground liquid argon TPC detector, a capable high resolution near detector, and a neutrino beam with initial proton beam power of $700 \mathrm{~kW}$ at $120 \mathrm{GeV}$, upgradable to $2.3 \mathrm{MW}$. 

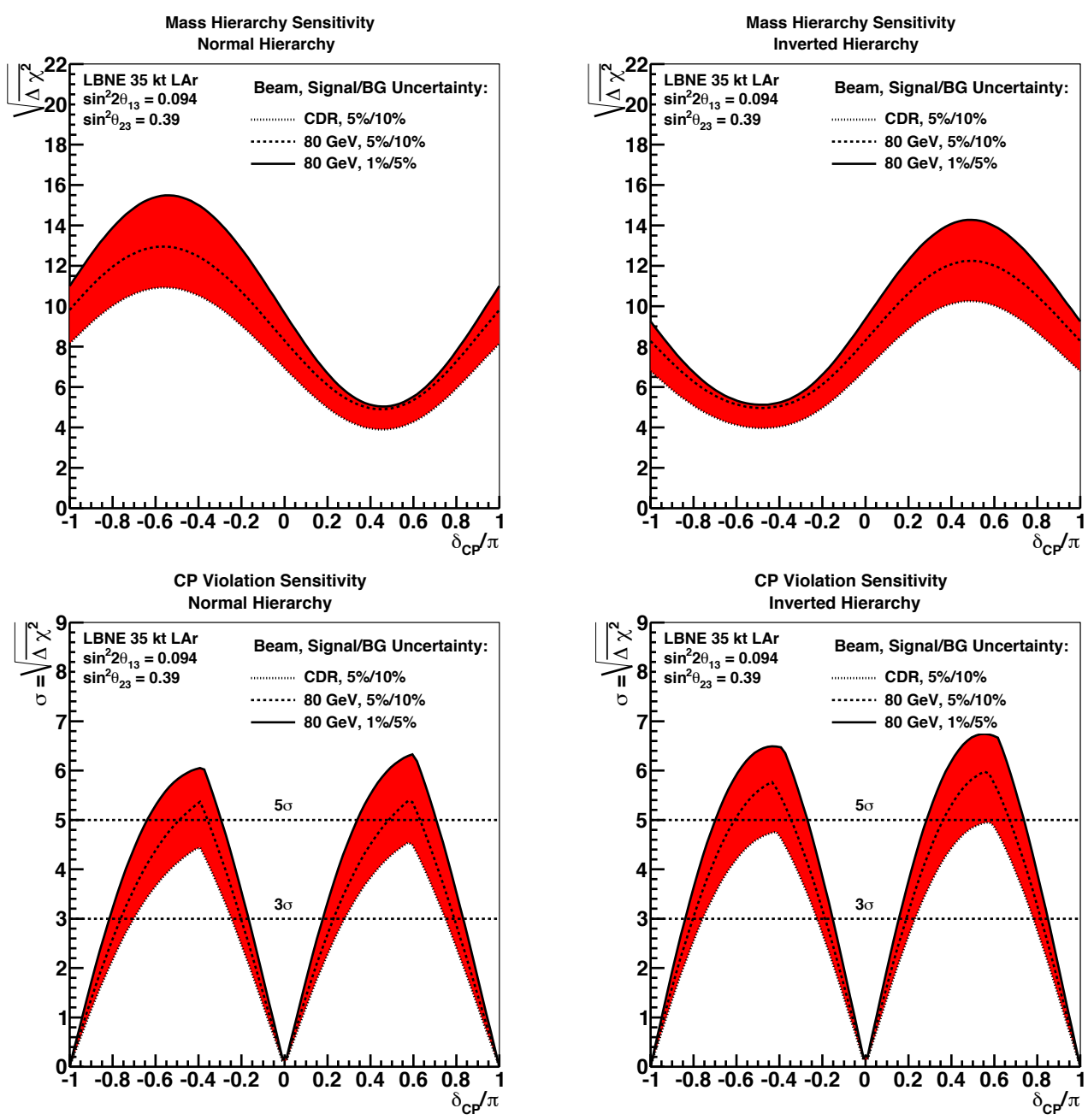

Figure 4: Sensitivity of LBNE with 35kt detector to the determination of mass hierarchy (top) and discovery of CP violation (bottom) for $\mathrm{NH}$ (left) and $\mathrm{IH}$ (right). The bands represent a range of possible sensitivities from variations in beam design and assumptions about the uncertainty of signal and background normalizations. For these plots the sensitivity is shown only for LBNE with beam data.

The first phase of LBNE with a $10 \mathrm{kt}$ detector has funding approval from the US DOE. The first phase, LBNE10, represents a significant improvement in sensitivity over current experiments and it could be significantly enhanced with international leadership. LBNE is building an international collaboration and embarking on a program of precision measurements which will produce exciting physics for decades to come.

\section{Acknowledgements}

This was prepared for the proceedings of the XV International Workshop on Neutrino Telescopes at the Istituto Veneto di Scienze, Lettere ed Arti in Venice held on March 11-15, 2013. The presentation was on behalf of the LBNE collaboration. This work was supported by the US Department of Energy under contract number DE-AC02-98CH10886. 

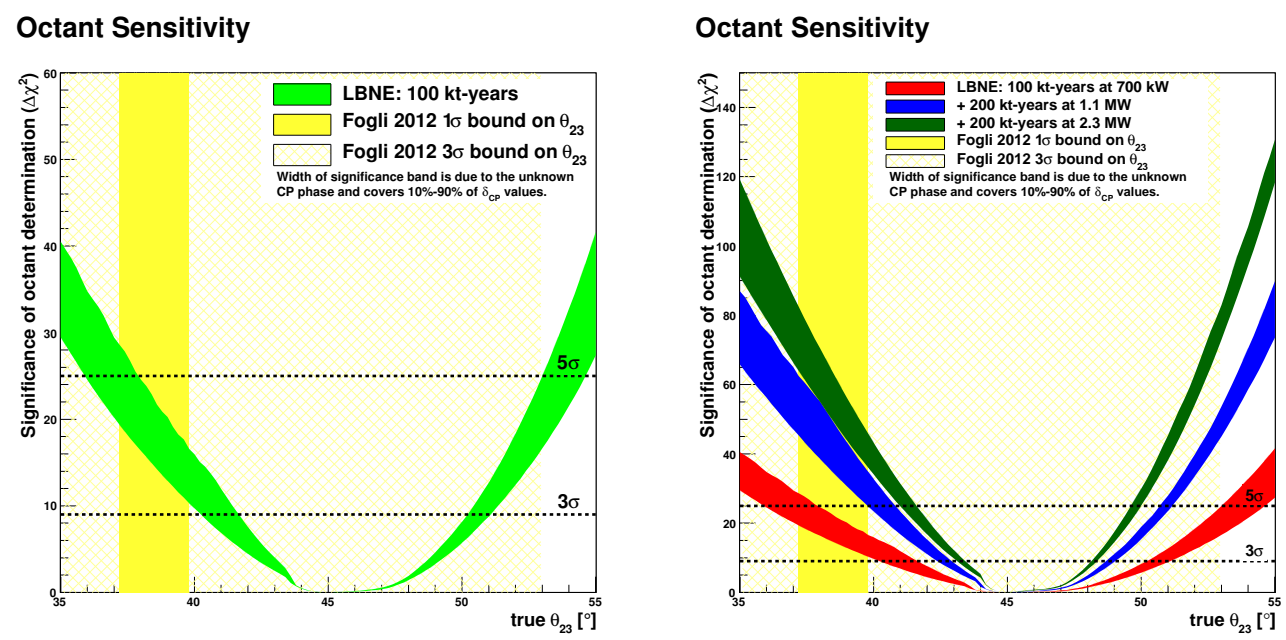

Figure 5: Sensitivity of LBNE10 (left) and higher exposures (right) to determination of the $\theta_{23}$ octant, as a function of the true value of $\theta_{23}$. The current $1 \sigma$ and $3 \sigma$ bounds on $\theta_{23}$ are shown.
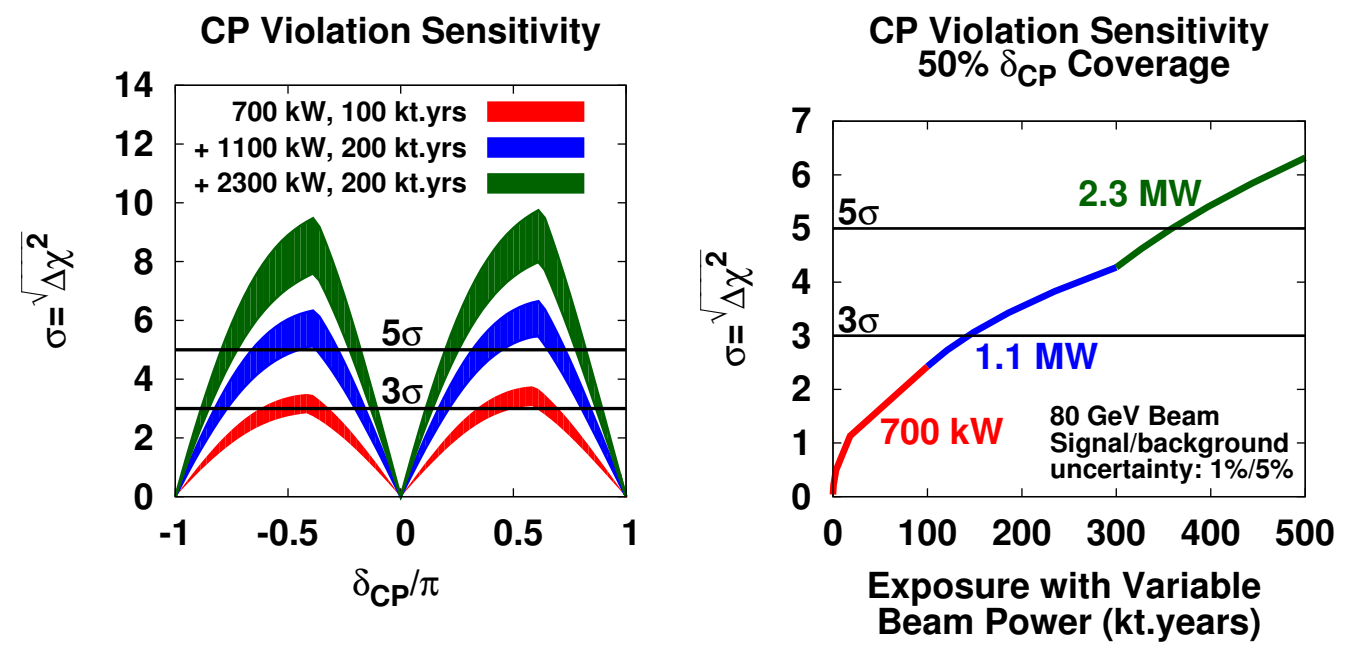

Figure 6: Left: Sensitivity of LBNE to discovery of CP violation, as a function of the true value of $\delta_{C P}$, for increasing exposure. The width of the bands represents a range of possible beam configurations. Right: Significance with which CP violation can be observed for $50 \%$ of possible $\delta_{C P}$ values, as a function of exposure in kt-years. This curve assumes the best beam configuration considered in the left panel.

\section{References}

[1] B. Pontecorvo, JETP 34, 172 (1958).

[2] Z. Maki, M. Nakagawa, and S. Sakata, Prog. Theor. Phys. 28, 870 (1962).

[3] J. Beringer et al. [Particle Data Group], Phys. Rev. D 86, 010001 (2012).

[4] F. P. An et al. [Daya Bay Collaboration], Phys. Rev. Lett. 108, 191802 (2012).

[5] F. P. An et al. [Daya Bay Collaboration], Chin. Phys. C 37, 011001 (2013).

[6] Y. Abe et al. [Double Chooz Collaboration], Phys. Rev. D 86, 052008 (2012). 
[7] J. K. Ahn et al. [RENO Collaboration], Phys. Rev. Lett. 108, 191802 (2012).

[8] P. Adamson et al. [MINOS Collaboration], Phys Rev. Lett. 110, 171801 (2013).

[9] K. Abe et al. [T2K Collaboration], Phys. Rev. D 88, 032002 (2013).

[10] P. Huber, M. Lindner, and W. Winter, Comput.Phys.Commun.167, 195 (2005).

[11] P. Huber et al., arXiv:hep-ph/0701187 (2007).

[12] LBNE Collaboration, Scientific Opportunities with the Long-Baseline Neutrino Experiment, 2013, $\operatorname{arXiV:1307.7335.~}$

[13] S. Holmes(ed.), Project X: Accelerator Reference Design, 2013, arXiv:1306.5022.

[14] A. Kronfeld and R. Tschirhart(ed.), Project X: Physics Opportunities, 2013, arXiv:1306.5009.

[15] G.L. Fogli et al., Phys. Rev. D86, 013012, arXiv:1205.5254 (2012). 2005 (2005).

[16] M. Apollonio et al., Eur. Phys. J., C27, (2003)331.

[17] M. Freund, Phys.Rev. D64 (2001) 053003; M. Freund, P. Huber, M. Lindner, Nucl.Phys. B615 (2001) 331-357;

[18] A. Cervera, et al., Nucl. Phy. B579(2000) 17.

[19] D.G. Michael et al., Nucl. Instrum. Meth. A596:190-228, 2008

[20] S. Kopp, Proc. 2005 IEEE Part. Accel. Conf., May 2005, Fermilab-Conf-05-093-AD and arXiv:physics/0508001.

[21] P. Adamson et al, Phys. Rev. D77, 072002 (2008).

[22] P. Adamson et al., Phys. Rev. Lett 101: 221804, 2008.

[23] H. Gallagher, Nucl. Phys. B (Proc. Suppl.) 112, 188 (2002); update at arXiv: 0806.2119 (2008).

[24] P. Adamson et al., Nucl. Inst. \& Meth. A556, 119 (2006).

[25] P. Adamson et al., Phys. Rev. Lett 101, 131802, 2008. 